\title{
Comparison of Incidence, Mortality and Treatment of Acute Myocardial Infarction in Hospitals in Japan and China
}

\author{
LIU ZHUO, TOMOKI HONMA* AND NOBUO KAKU* \\ Department of Emergency of Beijing 309 Hospital, Peking, 100091 China and \\ ${ }^{*}$ Critical Care Center of Kurume University Hospital, Kurume, 830 Japan
}

Recieved for publication October 6, 1992

\begin{abstract}
Summary: Acute myocardial infarction (AMI) is one of the most intractable diseases and is increasing rapidly in Japan and China. Two hospitals in Japan and China, the Critical Care Center of Kurume University Hospital and the Chinese Beijing 309 Hospital in China (abbreviated to Beijing 309 Hospital) were compared. The incidence, mortality and treatment of AMI were investigated in both hospitals from 1989 to 1991. The incidence of AMI for all patients admitted during the three years was $5 \%$ in Kurume University Hospital and $4.7 \%$ in Beijing 309 Hospital, which are similar rates. The average age of the patients in Beijing 309 Hospital was younger $(58 \pm 13)$ than in Kurume University Hospital $(64 \pm 11)$. The mortality rate in Kurume University Hospital was slightly lower than the rate in Beijing 309 Hospital $(8.1 \%$ vs $8.9 \%)$. Thrombolytic therapy was actively performed in both hospitals. In Kurume University Hospital, urokinase (UK:71.4\%) or recombinant tissue plasminogen activator (rt-PA: 28.6\%) was administered by intravenous $(85.7 \%)$ and intracoronary percutaneous transluminal coronary recanalization (PTCR:14.3\%) injection. In Beijing 309 Hospital, UK (32.7\%) or snake poison enzyme (SPE:62.3\%) was administered by intravenous $(85.8 \%)$ or intra-aortic $(14.2 \%)$ injection. Rt-PA was only used in Japan and SPE was only used in China, but both had very strong fibrinolytic effects and resulted in high success rates of coronary reperfusion. The incidence of direct coronary intervention with percutaneous transluminal coronary angioplasty (PTCA) and intra-aortic balloon pumping (IABP) for cardiogenic shock was much higher at Kurume University Hospital than at Beijing 309 Hospital. Though there were no differences in the use of coronary dilators, antiarrhythmics, analgesics and catecholamines in the two hospitals, Chinese herbal medicine was used in the Beijing 309 Hospital. In conclusion, the incidence of AMI was similar at both hospitals and the mortality rate was slightly lower at Kurume University Hospital than at Beijing 309 Hospital. Coupling the advanced technology applied in Japan and the Chinese herbal medicines used in China may advance the treatment of AMI.
\end{abstract}

Key words: acute myocardial infarction - recombinant tissue plasminogen activator-snake poison enzyme-Chinese herbal medicine-coronary care unit 


\section{Introduction}

Acute myocardial infarction (AMI) is a common disease, especially in western countries. It is increasing in eastern countries including Japan and China, thus management strategies for AMI have become important in both countries. The recent development of thrombolytic therapy markedly decreased the mortality for AMI( GISSI, 1986; Kawai, 1988).

The treatments and mortality rates for AMI during the past three years were compared in two hospitals in Japan and China. One is the Critical Care Center of Kurume University Hospital in Japan, and the other is Beijing 309 Hospital in China. Kurume University Hospital which was founded in 1928 has 1,200 beds and 21 specialities. The Critical Care Center was established in 1981 and has 35 beds including 5 beds for the coronary care unit (CCU). Beijing 309 Hospital is in Beijing, China, and was founded in 1952. It has 800 beds and 41 specialities. The department of emergency medicine has 23 beds, but no exclusive beds for the CCU. Though there are many differences between Japanese and Chinese medicine, the purpose of this report is to introduce new treatments for AMI in both countries.

\section{Materials and Methods}

The subjects included 160 patients with AMI in Kurume University Hospital and 248 patients in Beijing 309 Hospital during 1989-91. The diagnosis of AMI was based on a history of prolonged chest discomfort lasting at least $30 \mathrm{~min}$, after which a characteristic ST elevation in $\geqq 2$ leads and an elevation of serum levels of total creatine kinase (CK) and CK-MB isozyme occurred.

The incidence and mortality rates from AMI were investigated for all patients admitted to each hospital. The treatments of AMI were compared, including thrombolytic therapy, coronary dilators, vasopressors and artificial support for cardiogenic shock.

\section{Results}

1. Comparison of incidence and mortality rates for AMI in both hospitals (Table 1)

The average age of the 248 patients in Beijing 309 Hospital was younger ( $58 \pm$ 13) than in Kurume University Hospital $(64 \pm 13, \mathrm{P}<0.01)$. There were many more men than women in both hospitals. The incidence of AMI in all critical care patients was $5 \%$ in Kurume University Hospital and 4.7\% in Beijing 309 Hospital, which is similar. The mortality rate from AMI was $13 / 160$ (8\%) in Kurume University Hospital which was slightly lower than in Beijing 309 Hospital [22/248 (9\%)].

\section{Comparison of treatments in both hospi- tals (Table 2)}

Treatments using Swan-Ganz catheters (61\% vs $17 \%$ ) and intra-aortic balloon pumping (IABP, $8 \%$ vs $0 \%$ ) were more frequent in Kurume University Hospital than Beijing 309 Hospital. In Kurume University, dopamine and dobutamine were administered for cardiogenic shock and if these agents did not adequately elevate blood pressure, IABP was instantly initiated with respiratory management. Thrombolytic therapy was used in both hospitals. Though the frequency of PTCA was much higher in Kurume University Hospital (21\%) than Beijing 309 Hospital (1\%), thrombolytic therapy was more frequent in Beijing 309 Hospital (88\% vs 44\%). In Beijing 309 Hospital, thrombolytic agents were administered by intravenous (86\%) and intraaortic injections (14\%). In Kurume University Hospital, thrombolytic agents were administered by intravenous (86\%) and 
TABLE 1.

AMI patient profiles

\begin{tabular}{lcc}
\hline & Kurume University & Beijing 309 Hospital \\
\hline Number of patients & 160 & 248 \\
Pt. with AMI/total pt. & $160 / 3211(5.0 \%)$ & $248 / 5324(4.7 \%)$ \\
Age (years) & & \\
$\quad$ Mean \pm SD & $64 \pm 11$ & $58 \pm 13$ \\
$\quad$ Range & $30-87$ & $28-79$ \\
Sex & & \\
Male & 117 & 192 \\
Female & 43 & 56 \\
Mortalities & $13(8 \%)$ & $22(9 \%)$ \\
\hline
\end{tabular}

TABLE 2.

Comparison of treatment for AMI at Kurume University Hospital and Beijing 309 Hospital

\begin{tabular}{lcc}
\hline & Kurume University & Beijing 309 Hospital \\
\hline Number of AMI patients & 160 & 248 \\
Swan-Ganz catheter & $97(61 \%)$ & $42(17 \%)$ \\
IABP & $13(8 \%)$ & $0(0 \%)$ \\
PTCA & $33(21 \%)$ & $2(1 \%)$ \\
Thrombolytic therapy & $70(44 \%)$ & $217(88 \%)$ \\
Method & & \\
intravenous & $60(86 \%)$ & $186(86 \%)$ \\
intracoronary (PTCR) & $10(14 \%)$ & $0(0 \%)$ \\
intraaorta & $0(0 \%)$ & $31(14 \%)$ \\
Medicine & $50(71 \%)$ & $71(33 \%)$ \\
UK & $20(29 \%)$ & $0(0 \%)$ \\
Rt-PA & $0(0 \%)$ & $146(67 \%)$ \\
SPE & $160(100 \%)$ & $71(29 \%)$ \\
Coronary dilators & $0(0 \%)$ & $177(71 \%)$ \\
$\quad$ Nitroglycerin & & \\
pill of coronary dilatation & $160(100 \%)$ & $248(100 \%)$ \\
Antiarrhythmics & Dopamine & Dopamine \\
Lidocaine & Dobutamine & Dobutamine \\
Vasopressors & monkshood-ginseng \\
& morphine & morphine \\
Analgesics & Buprenorphine & Dolantin \\
\hline & & \\
& &
\end{tabular}


Before administration of $\mathrm{t}$-PA (1.5 hours after onset)

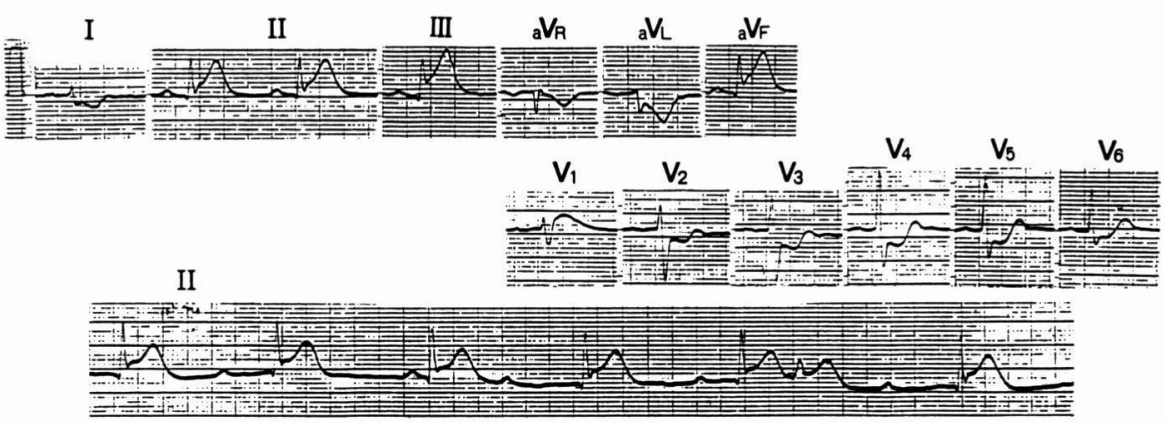

Immediately after administration of $\mathrm{t}-\mathrm{PA}$
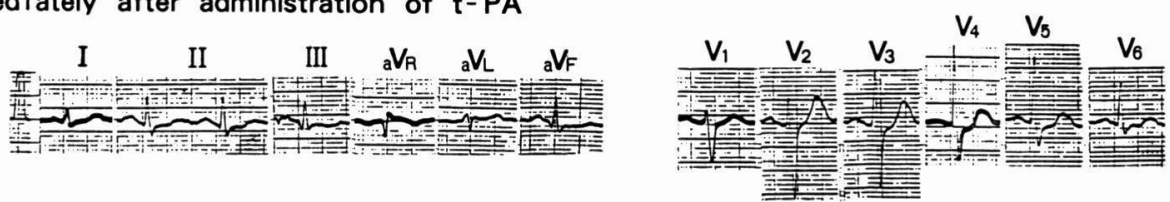

Fig. 1. A 64 year-old male. The ECG (upper) had ST elevation in II, III, $\mathrm{aVF}$, and complete atrio-ventricular block. After t-PA was administered, intravenously, the chest pain disappeared and the ECG (lower) had less ST elevation without the formation of an abnormal Q wave and the atrioventricular block also disappeared.

\section{Before administration of S.P.E (30min after onset)}

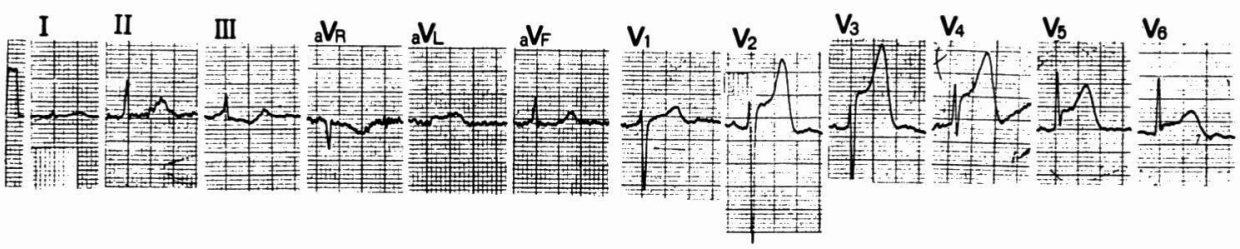

4 days after administration of S.P.E

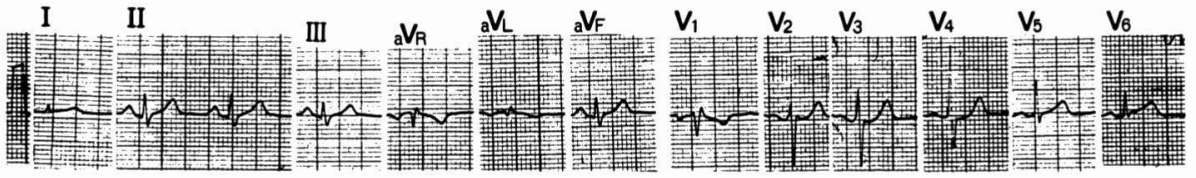

Fig. 2. A 64 year-old male. The ECG (upper) had ST elevation in I, $\mathrm{aV}_{\mathrm{L}}$, and $\mathrm{V}_{2}$ to $\mathrm{V}_{6}$. SPE was administered intraaortically followed by intravenous administration for three days. The ECG (lower) had less ST elevation without formation of an abnormal Q wave. 
intracoronary injections (PTCR, 14\%) within 6 hours after onset of AMI. The thrombolytics used were UK and SPE in Beijing 309 Hospital and UK (since 1990) and rt-PA (since 1991) in Kurume University Hospital. Thus the SPE was only used in China and rt-PA was only used in Japan. Nitroglycerin and calcium antagonists were routinely used as coronary dilators in Kurume University Hospital.

In Beijing 309 Hospital, $71 \%$ of the patients were treated by coronary dilation with Chinese herbal medicines. There were no differences in antiarrhythmics and analgesics used in the hospitals. In Beijing 309 Hospital, Chinese herbal medicines were frequently used to dilate coronary arteries and improve blood viscosity.

Figs 1 and 2 show results from representative cases using rt-PA in Kurume University Hospital and SPE in Beijing 309 Hospital.

\section{Discussion}

The incidences of AMI were similar in the Chinese and Japanese hospitals. But the average age of the patients with AMI in Beijing 309 Hospital was less than that for Kurume University Hospital. The reason why the Chinese patients were younger may be based on the diet or management of coronary risk factors. Japanese eat more fish than the Chinese, which may prevent coronary artery disease. Japanese doctors aggressively treat hypertension, diabetes mellitus, hypercholesterolemia as risk factors for ischemic heart disease.

The management for coronary intervention of $A M I$ and cardiogenic shock with PTCR, PTCA, IABP in Kurume University was much more advanced than at Beijing 309 Hospital. This is characteristic of the advanced technology in Japan and may play a role in the reduced mortality from AMI. If Kurume University can educate and financially aid Chinese doctors, Chinese medicine will develope more rapidly in the future.

In Kurume University Hospital, thrombolytics were administered by intravenous or intracoronary (PTCR) injection and in Beijing 309 Hospital, they were administered by intravenous or intra-aortic injection. The technique of intra-aortic injection requires a special catheter which has no end hole but has two side holes located $1 \mathrm{~cm}$ from the tip of the catheter. After the catheter is inserted in the femoral artery and localized just above the aortic valve where the coronary ostium opens, UK or SPE is injected. Further examination is needed to support the effectiveness of this technique.

Since 1991, intracoronary thrombolytic therapy and intravenous administration of rt-PA to patients with AMI has become widely available in Japan. A thrombolysis in myocardial infarction (TIMI) study showed that intravenous thrombolysis with rt-PA led to successful coronary reperfusion in $85 \%$ of patients, which was higher than with UK (TIMI group, 1989). Rt-PA has a high affinity for plasminogen in thrombi. So rt-PA activates the plasminogen in the thrombus to plasmin without decreasing the plasma level of fibrinogen and plasminogen (Matsuda, 1992). In Kurume University, 20 patients admitted within 6 hours after the onset of an AMI were treated with rt-PA and 17 of the 20 patients (85\%) had a successful coronary reperfusion (Fig. 1). The most common side effect of rt-PA was minor bleeding, however fatal reperfusion arrhythmias did occur immediately after successful reperfusion at a frequency of less than $5 \%$. SPE is an activator of thrombolysis and consists mainly of argin lipase which is extracted from snake poison. SPE reduces fibrinogen in plasma but can aggregate platelets (Wang, 1987). SPE was administered by intravenous or 
intra-aortic injection for one day followed by continuous administration for 15 to 20 days. The side effects of SPE were bleeding and hypersensitivity.

In Kurume University Hospital, nitroglycerin and calcium antagonists were routinely used as coronary dilators. Nitroglycerin can improve cardiac contractions by reducing ventricular loading, altering the myocardial oxygen supply-demand ratio and redistributing blood flow to ischemic zones (Cintron et al. 1988). Calcium antagonists also dilate coronary arteries and protect against coronary spasm (Hill et al. 1983).

In recent years, a lot of Chinese herbal medicine was used for treatment of AMI in China. Medications for coronary dilatation consist of toad cake herneol which dilates coronary arteries like nitroglycerin and salivary miltiorrhiza which increases blood volume in the coronary artery and decreases blood viscosity like Dextran (Bai, 1987). The monkshoodginseng was used to elevate blood pressure and induce positive inotropic effects like dopamine. These Chinese herbal medicines have adequate efficacy to treat AMI without evident side effects.

In conclusion, the incidence of AMI was similar in both hospitals and the mortality was slightly lower in Kurume University Hospital. Though standard management strategies for AMI were similar in both hospitals, advanced technologies, such as PTCA and artificial support like IABP were used more in Kurume University Hospital. Chinese herbal medicine which was safe and effective was commonly used in Beijing 309
Hospital. Coupling the advanced technology actively applied in Japan with Chinese herbal medicines used in China may further enhance treatment for AMI.

\section{References}

BAI, L.Z. (1987). Salivia Miltiorrhiza. In $D i$ rection of Clinical Medicine, pp 501. Peking: China Anhwen Publish Company.

Cintron, G. B., Glasser, S.P., Weston, B. A., Linares, E., Conti, C.R. et al. (1988). Effect of intravenous isosorbide dinitrate versus nitroglycerin on elevated pulmonary arterial wedge pressure during acute myocardial infarction. Am. J. Cardiol. 61, 21-25.

Gruppo Italiano Per Lo Studio Della Streptochinasi Nell' Infarto Miocardico (GISSI). (1986). Effectiveness of intravenous thrombolytic treatment in acute myocardial infarction. Lancet 1, 397-401.

Hill, J. A., Feldman, R. L., Conti, C. R., Hill, C. K. and Pepine, C.J. (1983). Long term responses to nifedipine in patients with coronary spasm who have an initial favorable response. Am. J. Cardiol. 52, 26-29.

KawAI, C. (1988). Advances in intracoronary thrombolysis treatment. J. Cardiol. 18, 11491153.

Matsuda, T. (1992). In each thrombosis position and indication of thrombosis therapy. Prog. Med. 12, 163-167.

The TIMI study group. (1989). Comparison of invasive and conservative strategies after treatment with intravenous tissue plasminogen activator in acute myocardial infarction. - Results of thrombolysis in myocardial infarction. (TIMI) phase II trial. N. Eng. J. Med. 320, 618-627.

W ANG, J.Z. (1987). Thrombin-like enzyme. In Direction of Clinical Medicine, pp. 878. Peking: China Anhwen Publish Company. 